\title{
Vie de la recherche
}

\section{Le comité scientifique «Sociétés, environnement et développement durable » du programme Environnement, vie et sociétés du CNRS (1999-2002) : une esquisse de bilan}

\author{
Franck-Dominique Vivien ${ }^{1}$, Tatiana Muxart ${ }^{2}$ \\ 1 Économiste, Université de Reims Champagne-Ardenne, UFR Sciences économiques et gestion, Laboratoire «Organisations \\ marchandes et institutions », 51096 Reims, France \\ 2 Géographe, CNRS, UMR8591 Laboratoire de géographie physique, 92195 Meudon, France
}

Mots-clés :

politique scientifique;

interdisciplinarité;

programme

Environnement

du CNRS

\section{Keywords:}

science policy;

interdisciplinarity;

CNRS Environment

Program
Résumé - Nous esquissons un bilan du travail réalisé par le comité scientifique «Société, environnement et développement durable » (1999-2002), instance de programmation du PEVS-2 du CNRS. Nous présentons ses principaux résultats scientifiques, la structuration des communautés scientifiques à laquelle il a contribué, mais aussi les difficultés institutionnelles inhérentes à la politique scientifique relative à l'environnement et au développement durable.

\begin{abstract}
The scientific committee "Society, Environment and Sustainable Development" of the CNRS Program Environment, Life and Society: overview of results. Our paper summarizes work carried out by the scientific committee "Society, Environment and Sustainable Development" (1999-2002), a programming instance of CNRS PEVS-2. We present its main scientific results, the structuring of scientific communities to which it contributed, and the institutional difficulties inherent in science policy dealing with the environment and sustainable development.
\end{abstract}

Dernier avatar d'une programmation interdisciplinaire qui s'est poursuivie pendant plus de vingt ans, le programme Environnement, vie et sociétés du CNRS (dit PEVS-2 à partir de 1999) a cessé d'être renouvelé en $2003^{3}$. Huit ans après, au moment où le CNRS connaît une réforme majeure aboutissant à sa restructuration en dix instituts pluridisciplinaires, dont l'Institut Écologie et environnement (INEE) aux thématiques s'apparentant à celles du PEVS, il nous a semblé intéressant de présenter le bilan de l'une des instances de programmation de l'ex-PEVS-2, le comité scientifique "Sociétés, environnement et développement durable » (SEDD). Héritier d'un travail de réflexion mené au sein de comités scientifiques antérieurs, SEDD avait pour finalité de promouvoir une recherche interdisciplinaire sur l'environnement associant des chercheurs des sciences de l'homme et de la société (SHS) et des chercheurs des sciences de la vie (SDV) et des sciences de l'Univers (SDU). Or, cette am-

Auteur correspondant : F.-D. Vivien, fd.vivien@univ-reims.fr

${ }^{3}$ L'historique de ce programme a été retracé par Jollivet (2001), Pavé (2001) et Beck et al. (2008). bition, qui ne va pas de soi, a rencontré des difficultés conceptuelles et opérationnelles. Les débats, à la croisée du scientifique et de l'institutionnel, qui ont abouti à la disparition du PEVS-2, sont, en effet, les mêmes qu'aujourd'hui. En particulier, la question reste posée de la place dévolue aux SHS dans le traitement des questions d'environnement et de développement durable.

\section{L'action du comité SEDD et sa participation à la politique générale du PEVS-2}

\section{Les objectifs et la démarche de SEDD}

Le comité SEDD, dont les deux auteurs de cet article ont été responsables, a produit des travaux qui sont, pour une part, la poursuite des actions et réflexions conduites par deux comités scientifiques du PEVS-1 (1995-1998) : «Environnement, sociétés et développement à long terme » (ESDLT) et « Sociétés, environnement et actions de l'homme» (SEAH). 
Le premier était composé uniquement de représentants des SHS, ce qui était a priori paradoxal au sein d'une institution programmatique souhaitant recouper tous les champs scientifiques. La raison en est que les chercheurs des SHS se montraient alors réticents à intervenir sur des questions environnementales qui ne faisaient pas partie de leurs interrogations disciplinaires habituelles. Dans ce contexte, la mission de ESDLT était d'intéresser, puis d'inciter les équipes des SHS à s'engager dans des projets de recherches sur l'environnement. Il s'agissait de constituer et de fidéliser un vivier d'équipes habituées à travailler ensemble (interdisciplinarité intra-SHS) et prêtes à s'associer aux chercheurs des sciences de la nature, en ayant leurs propres points de vue sur les questions traitées.

Le comité SEAH avait, à l'opposé, une vocation transdisciplinaire. Il visait à aborder les thématiques environnementales en dépassant les seules approches de l'écologie et des sciences de la nature pour les resituer dans le cadre plus large des interactions hommes-milieux et de leurs évolutions historiques.

Le comité SEDD du PEVS-2 a été organisé avec l'idée d'une certaine continuité thématique avec ESDLT. Sa priorité a été de promouvoir une interdisciplinarité large des SHS, tout en modifiant la hiérarchie des disciplines qui prévalait précédemment. ESDLT, qui était présidé par un économiste, avait été dominé par les chercheurs de cette discipline. Sans négliger cette dernière, notre principal souci a été de disposer de l'éventail le plus large possible des SHS, avec des archéologues, historiens, anthropologues, géographes physiciens et humains, juristes, sociologues et politistes. Nous avons souhaité également une ouverture vers des disciplines qui relèvent des sciences de l'ingénieur (l'agronomie, notamment). Cette double perspective rattachait aussi clairement SEDD aux finalités et à l'action poursuivies antérieurement par le comité SEAH. En outre, afin qu'un apprentissage se fasse et qu'un héritage se transmette, SEDD comprenait des «anciens », des chercheurs ayant déjà une bonne expérience des pratiques interdisciplinaires développées au CNRS ou au sein d'autres organismes de recherche (département Sciences pour l'action et le développement [SAD] de l'Inra, notamment), et des «nouveaux » qui démarraient dans la carrière scientifique et s'étaient récemment lancés dans ce type d'aventure ${ }^{4}$.

\footnotetext{
${ }^{4}$ Le comité SEDD comprenait : Franck-Dominique Vivien (chargé de mission SHS, économie), Tatiana Muxart (présidente, géographie physique), Paul Arnould (biogéographie), Corinne Beck (histoire, archéologie), Maryvonne Bodiguel (sociologie politique), Jean-Paul Bravard (géographie physique), Joëlle Burnouf (histoire, archéologie), Yann Laurans (économie), Yves Luginbühl (géographie), Anne Luxereau (anthropologie), Michel Magny (paléoclimatologie), Sandrine Maljean-Dubois (droit), François Papy (agronomie), Bernard Picon (sociologie), Girolamo Ramunni (histoire des sciences), Marie Roué (anthropologie), Jean-Michel Salles (économie),
}

Tout en poursuivant dans la voie tracée par ESDLT et SEAH et en s'inscrivant dans la politique générale du PEVS-2, SEDD a défini ses propres priorités en se concevant comme un incubateur d'idées, de concepts et de questionnements nouveaux sur l'environnement et le développement durable. Cette capacité d'ouverture a été favorisée par la large gamme d'outils de programmation scientifique dont il disposait : appels d'offres, journées d'étude, séminaires, colloques, écoles thématiques... Le rôle de SEDD a été de savoir en jouer, en fonction du degré de structuration de la communauté de chercheurs susceptibles de porter telle ou telle question de recherche et de la maturation de celle-ci.

\section{L'intégration de l'action du comité SEDD dans la politique générale du PEVS-2}

$\mathrm{Au}$ début de son fonctionnement, le PEVS-2 est apparu comme une structure multipolaire, avec relativement peu d'interaction entre ses comités scientifiques ${ }^{5}$, chacun étant constitué autour d'un chargé de mission représentant un département scientifique du CNRS. Cela n'a pas empêché plusieurs membres du comité SEDD de coopérer à certaines actions lancées par les autres comités du PEVS-2 (par exemple, co-organisation d'une école thématique sur la prospective ou réflexion sur les concepts et les outils d'observation de l'environnement, à moyenne échelle et sur le long terme). Dans la seconde partie du mandat du PEVS-2, ces différentes programmations ont convergé grâce au programme de labellisation des «Zones Ateliers» (ZA), autour duquel une structure de programmation « inter-comités » et «inter-chargés de mission » a été mise sur pied. À l'arrière-plan de ces outils que constituent les ZA se trouve la notion d'anthroposystème.

\section{La notion d'anthroposystème}

L'approche systémique a été utilisée d'emblée par l'écologie, avec les concepts d'écosystème, puis d'écocomplexe, et plus tardivement par la géographie, avec la notion de géosystème. La convergence de bilans conceptuels entre ces deux disciplines, travaillant à l'intersection du naturel et du social, et la nécessité, à travers la démarche systémique, de relancer l'interdisciplinarité ont été soulignées dans Les Passeurs de frontières (Jollivet, 1992). C'est dans le prolongement de ces réflexions que les membres du PEVS-2 (Lévêque et al., 2003) ont contribué à la définition de la notion d'anthroposystème :

Sander van der Leeuw (archéologie), Bruno Villalba (sciences politiques).

5 Outre SEDD, relevant du département SHS, deux autres comités ont fonctionné : «Écosystèmes et environnement » (C3E) et «Modélisation, transferts d'informations, valorisation pour l'environnement » (MOTIVE), liés respectivement aux départements SDV et SDU. 
« système interactif entre deux ensembles constitués par un (ou des) sociosystème(s) et un (ou des) écosystème(s) naturel(s) ou artificialisé(s) s'inscrivant dans un espace géographique ${ }^{6}$ donné et évoluant avec le temps ». L'accent est mis sur les interactions et co-évolutions entre les sociétés et les milieux. Dans le cadre des débats relatifs à la programmation scientifique et du contexte institutionnel très défavorable de l'époque (nous y reviendrons), la notion d'anthroposystème a pris une visée stratégique, en offrant un référentiel dans lequel pouvaient s'inscrire clairement les SHS face à la tendance hégémonique des sciences de la nature. L'utilisation de cette notion se veut aussi opératoire : elle vise à peser sur les pratiques de recherche-notamment dans le cadre des ZA - en proposant une notion intégratrice, une unité d'étude permettant les confrontations de regard et d'analyse.

\section{Les Zones Ateliers}

S'inspirant des «Long Term Ecological Research », un réseau national d'observatoires écologiques mis en place aux États-Unis, l'objectif du programme ZA (Lévêque et al., 2000) est de comprendre et de modéliser le fonctionnement des anthroposystèmes, de caractériser les trajectoires co-évolutives des systèmes naturels et sociaux qui les composent et d'établir des scénarios, à caractère prospectif, des divers futurs possibles. La démarche ZA s'inscrit d'emblée dans une problématique de développement durable, ce dernier étant fondé sur un usage et un partage à long terme des ressources, des espaces et des territoires. Outre la priorité donnée à l'étude des modes de gestion intégrée des ressources et des milieux, l'approche s'intéresse à l'analyse des modalités des systèmes de gouvernance. Considérées comme des lieux d'observation et d'expérimentation de politiques d'aménagement et de gestion du territoire, les $\mathrm{ZA}$ représentent un moyen très intéressant d'inclure les SHS dans la définition des problématiques environnementales.

Au plan opératoire, cette approche vise à mettre en place des outils conceptuels, méthodologiques et informationnels permettant d'articuler le local, le régional et le global ; le passé, le présent et le futur ; le court, le moyen et le long terme et enfin l'économique, le social, le technique et l'écologique. La pérennisation de l'acquisition de données écologiques et sociales (notamment au sein de sites ateliers instrumentés inclus dans la ZA), leur informatisation et leur mise à disposition de la communauté scientifique sont jugées essentielles. Il en est de même de la nécessité de mise en réseau des ZA aux échelles nationale et européenne.

\footnotetext{
6 «Espace géographique » est un terme général qui doit être compris au sens d'unité territoriale, plus ou moins vaste, occupée, utilisée, exploitée et transformée par un individu, un groupe social ou, plus généralement, par une société humaine au cours du temps.
}

Les ZA ont fait l'objet d'un appel d'offres du PEVS-2, lancé en 2000, puis renouvelé ${ }^{7}$. Le comité scientifique ZA était présidé par Alain Pavé, ancien directeur du PEVS-1. Outre les membres de l'équipe dirigeante du PEVS-2 (le directeur adjoint, Christian Lévêque et les chargés de mission), ce comité comprenait des chercheurs des trois autres comités scientifiques du programme, ainsi que des scientifiques choisis pour leur compétence et leur expérience dans la conduite de recherches pluridisciplinaires sur l'environnement. Le rôle des membres de SEDD a été de prôner l'implication systématique des SHS lors de la labellisation des ZA dirigées par les chercheurs naturalistes, voire de proposer des équipes locales SHS lorsque celles-ci manquaient. Enfin, le lancement par SEDD, en 2002, de 1'appel d'offres «La gouvernance en question » a eu notamment pour but de renforcer la présence des questions et des équipes des SHS au sein des ZA déjà créées.

\section{La programmation scientifique du comité SEDD}

La politique mise en œuvre par SEDD peut être présentée sous la forme d'une exploration de trois temporalités : le passé, avec l'histoire des interactions sociétésmilieux, l'actuel, avec l'étude des politiques d'environnement et de développement durable mises en œuvre, en particulier dans le domaine de la biodiversité, et le futur, avec l'accent mis sur la prospective dans le domaine de l'environnement. Dans ce qui suit, nous avons choisi de présenter les apports scientifiques obtenus pour les deux premiers axes de recherche. Pour chacun d'entre eux, nous indiquerons les enseignements généraux qui peuvent être tirés d'une approche interdisciplinaire (interne aux SHS ou en association avec les sciences naturelles) des questions traitées.

\section{Les interactions des sociétés avec leurs environnements dans la longue durée}

Plusieurs actions de recherche complémentaires ont été lancées sur ce thème : l'appel d'offres « Histoire des interactions sociétés-milieux », ainsi qu'une animation scientifique reposant, d'une part, sur un séminaire de réflexion autour de la notion de "crise environnementale » qui s'est déroulé sur un peu plus de deux ans et, d'autre part, sur deux colloques intitulés « Les fleuves aussi ont une histoire », qui se sont tenus en juin 2001 et avril 2002.

\footnotetext{
${ }^{7}$ Quatorze ZA ont été labellisées avant la suppression du PEVS-2. Malgré les efforts des représentants de SEDD au sein du comité ZA, la plupart étaient dirigées par - et comprenaient surtout - des chercheurs des sciences naturelles.
} 
Appel d'offres « Histoire des interactions sociétés-milieux »

La première action lancée par SEDD a été conçue dans la continuité du travail effectué par le comité ESDLT qui, au terme de séances de séminaire organisées en 19971998, avait mis l'accent sur la nécessité d'explorer plus avant les interactions, sur le long terme, entre les sociétés et leurs environnements. Cet appel d'offres visait tout d'abord à mieux structurer, élargir et rendre "visibles", dans leurs propres disciplines, les communautés d'historiens et d'archéologues acceptant de travailler avec d'autres spécialistes sur les thématiques environnementales. Il s'agissait ensuite d'inciter les équipes à mener une réflexion interdisciplinaire afin de tenter de répondre à deux interrogations ${ }^{8}: \mathrm{d}^{\prime}$ une part, en quoi l'étude des phénomènes sociaux et naturels ayant affecté la dynamique d'un anthroposystème dans son passé permet-elle de mieux comprendre ses transformations ultérieures et son fonctionnement actuel? D'autre part, dans un contexte d'incertitude scientifique, quels sont les éléments tirés de l'histoire qui peuvent éclairer et aider la prise de décision concernant l'évolution souhaitée des rapports futurs d'une société avec son milieu?

Comme pour tout appel d'offres, les réponses retenues portaient sur des thèmes, des espaces et des temps historiques variés. Elles correspondaient soit à des programmes élaborés, fruits d'un premier travail collectif pluridisciplinaire, soit à des propositions conjoncturelles de chercheurs escomptant un financement pour lancer une nouvelle étude. Une sensibilisation des chercheurs à l'approche interdisciplinaire a eu lieu au cours de deux journées d'animation et de suivi du programme, en octobre 2001 et 2002.

Des enseignements d'ordre général peuvent être tirés de ces travaux. Dans le domaine de la recherche des types de causalités, naturelles et/ou sociales, à l'origine des transformations et de l'évolution à long terme des anthroposystèmes étudiés, les explications unicausales ont été le plus souvent rejetées. L'intérêt d'une approche interdisciplinaire large (c'est-à-dire englobant SHS et sciences de la nature) a permis, entre autres, d'éviter la focalisation sur un seul «type » de causalités, d'origine sociale ou, à l'opposé, de source naturelle, selon que l'équipe était composée d'une majorité de chercheurs des SHS ou bien des SDV et SDU.

L'imbrication synchrone des causalités de nature différente et/ou leur succession dans le temps, au sein d'un

\footnotetext{
${ }^{8}$ Notons que ces questionnements à caractère interdisciplinaire traversent des interrogations disciplinaires plus classiques : par exemple, la validité scientifique du croisement des sources (textuelles et/ou sédimentaires) d'origine, de qualité et de fiabilité variables, ou les choix méthodologiques de l'étude des «environnements » passés, concept inconnu, est-il besoin de le rappeler, dans les textes anciens.
}

même ou de divers niveaux d'organisation constituant les systèmes naturels et sociaux, ont été clairement mises en évidence. La complexité des situations est, à cet égard, quasiment toujours la règle. Soulignons que cette capacité à démêler l'écheveau des enchaînements spatiotemporels des causalités naturelles et/ou sociales, de clarifier les dynamiques complexes d'autorégulation qui en découlent et d'appréhender au mieux les changements passés et actuels affectant un anthroposystème, nécessite, à la fois, une démarche interdisciplinaire large et une approche sur le long terme. Seule cette dernière autorise une appréhension cinématique des déterminismes à l'origine des processus de transformations brutales ou progressives d'un système. À l'opposé, une étude des phénomènes à court terme, notamment l'analyse du seul fonctionnement actuel d'un système, risque de s'apparenter à une sorte « d'arrêt sur image » de la dynamique globale ne donnant qu'une "vision » tronquée des évolutions en cours et conduisant à occulter celles qui apparaissent «invisibles », car trop lentes pour pouvoir être saisies dans leurs effets. En particulier, la connaissance et la prise en compte des héritages naturels et sociaux (cf. infra et note 11) sont essentielles pour déterminer le fonctionnement actuel de l'anthroposystème et évaluer ses capacités de résilience et d'adaptation aux changements en cours ou probables dans un futur proche. Cette approche interdisciplinaire et historique a conduit bien souvent les équipes à déconstruire le «problème environnemental » considéré au départ de l'étude comme étant un fait «objectif» et à le reconstruire dans une perspective centrée sur les dynamiques de co-évolution des systèmes naturels et sociaux. Une telle approche est certainement mieux armée que ne l'est la seule démarche actualiste pour proposer des scénarios prospectifs crédibles des transformations à venir de ces systèmes hybrides.

\section{Temps et espaces des crises sur l'environnement}

Procédant également de l'héritage du comité ESDLT, ce séminaire, qui a rassemblé des archéologues, historiens, géographes, paléo-environnementalistes, anthropologues, sociologues, juristes et politistes, avait pour objectif d'engager un dialogue interdisciplinaire sur les interactions à caractère paroxysmique des sociétés avec leurs milieux au cours de leur histoire. Les travaux ont été circonscrits à la crise des ressources renouvelables dans l'espace rural ou marin.

De façon générale, plus que la question de la rareté (naturelle ou par surexploitation) de la ressource, ce qui induit une crise relève le plus souvent de l'organisation sociale et de la place des acteurs dans les dispositifs de régulation. Ces crises peuvent être déclenchées par les modifications du statut et du mode de gestion de ces ressources. Les normes et règles juridiques sont parfois des facteurs de la crise, voire la crise elle-même. La crise n'est donc pas réductible au 
seul dysfonctionnement biophysicochimique d'un environnement, elle naît à l'intersection des systèmes naturels et sociaux. Elle apparaît lorsque le seuil de rupture dans l'acceptabilité sociale des changements est franchi et que la résilience de l'anthroposystème ne semble plus assurée. Là, comme précédemment, les recherches remettent en cause l'unicité causale, naturelle ou sociale, au profit de l'imbrication spatiotemporelle de causes diverses, qui se succèdent ou interfèrent entre elles, à différents niveaux d'organisation spatiale de l'anthroposystème. Par ailleurs, elles soulignent le rôle fondamental joué par les représentations sociales de la nature dans l'action politique. Divers référents et modèles culturels organisent ces représentations au sein desquelles ils se superposent et se recomposent sans s'exclure, même lorsqu'ils sont contradictoires. Il en découle l'existence de perceptions de la nature opposées au sein d'un même groupe social, voire d'un même individu, selon la prévalence du point de vue adopté. Les acteurs sociaux qui mettent en scène la crise et les décideurs qui la régulent dans le cadre de l'action publique ont tendance à choisir les représentations qui servent leurs intérêts matériel et idéologique. Le rôle joué par les grands corps d'ingénieurs d'État (Eaux et Forêts, Ponts et Chaussées) dans la gestion des crises a été relevé. Il tend à privilégier le savoir technique sur le savoir scientifique et pose, de façon plus générale, la question de la hiérarchie entre les différents types de savoirs (y compris le savoir profane) à des fins de règlement des crises.

\section{Les fleuves aussi ont une histoire}

Le premier colloque, qui a rassemblé des paléoenvironnementalistes (géologues du Quaternaire, géomorphologues, palynologues, malacologues...) et des archéologues, a permis la reconstitution des variations paléohydrologiques en France depuis 15000 ans et leur liaison avec les oscillations du climat et les changements successifs de l'utilisation des milieux fluviaux et lacustres par les sociétés à partir du Néolithique. Les résultats ont permis d'établir une comparaison et une synthèse des causes et des processus à l'œuvre dans les transformations affectant les rivières et lacs, d'une part, au Tardiglaciaire, lorsque les déterminismes naturels ${ }^{9}$ sont prévalents et, d'autre part, à l'Holocène, période où se superpose, de façon de plus en plus prégnante, la pression exercée par les activités humaines. Les synchronismes ou les décalages temporels dans le déroulement des dynamiques et les effets des processus naturels entre eux et/ou des processus anthropiques ont été précisés. Au plan cognitif, l'appellation «impacts anthropiques » 10

\footnotetext{
9 D'ordre climatique, mais aussi géologique, géomorphologique et écologique.

10 Cette appellation est surtout privilégiée par les chercheurs des sciences de la nature.
}

est apparue impropre, car trop englobante et pas assez précise. Elle doit se décliner via l'étude du groupe social (ou de la société, selon l'échelle) et des traits qui le (ou la) caractérisent (modes de vie, comportements et référents culturels, pratiques et modes de gestion de l'espace, potentialités techniques et capacités adaptatives aux changements naturels ou induits par ses propres activités, etc.).

Le second colloque, qui a rassemblé des historiens, des archéologues, des géographes physiciens et plusieurs autres spécialistes des sciences sociales, traitait de la période préindustrielle. La réflexion a porté, en premier lieu, sur des questions méthodologiques. En particulier, les erreurs d'interprétation dues à une utilisation sans précaution ni critique des sources écrites et cartographiques anciennes par des non-historiens ont été dénoncées avec vigueur. L'approche interdisciplinaire est à cet égard seule garante du croisement le plus objectif possible des archives textuelles et sédimentaires. Dans certains cas favorables, elle a permis, par emprunt d'une démarche utilisée en biogéochimie, de proposer une modélisation «théorique » plausible des bilans biophysicochimiques passés, consécutifs à l'utilisation par un groupe social de son environnement. Par ailleurs, l'étude des pratiques sociales et des modes de gestion des espaces hydrauliques conduit à poser la question des risques et, en corollaire, celle des héritages tant naturels que sociaux. Les risques liés aux espaces fluviaux ne sont pas réductibles aux seuls effets des aléas naturels, ils peuvent aussi résulter des aménagements antérieurs qui, à leur tour, induisent de nouvelles vulnérabilités et de nouveaux dangers ${ }^{11}$. Par ailleurs, les travaux ont montré qu'en raison du nombre de lacunes dans le corpus des données, plusieurs interprétations possibles du fonctionnement passé d'un anthroposystème pouvaient être proposées sous forme de scénarios rétrospectifs, à l'image de ceux imaginés en prospective, les chercheurs indiquant celui qu'ils pensent être le plus plausible dans l'état actuel des recherches.

\section{Biodiversité : de la définition de l'objet à sa gestion}

À l'époque où l'Institut français de la biodiversité n'existait pas encore, le PEVS-1 finançait des recherches sur la diversité biologique. Le PEVS-2 a poursuivi cette politique, en mettant l'accent sur les recherches en SHS. L'intérêt pour cette thématique était, il est vrai, fort grand de la part de son directeur adjoint, C. Lévêque (1998), écologue impliqué dans l'expertise internationale en matière de diversité biologique. Plusieurs actions ont donc

\footnotetext{
11 Ainsi, certains risques naturels actuels (inondations des habitations construites dans le lit majeur ou sur le littoral, à l'arrière des digues ; déstockage des polluants d'origine industrielle, etc.) sont en fait hérités des modes passés d'organisation, d'utilisation et d'aménagement de l'espace et des ressources.
} 
été lancées par SEDD : une école thématique du CNRS sur «Biodiversité : quelles recherches à l'intersection des SDV et des SHS » ; des journées d'étude sur « Biodiversité et appropriation : les droits de propriété en question » et un séminaire «Biodiversité et ONG».

\section{Biodiversité : quelles recherches à l'intersection des SDV et SHS?}

L'objet « biodiversité » peut à la fois se définir comme un élément rhétorique, un concept scientifique et une catégorie juridico-administrative. Si son efficacité joue simultanément sur ces trois registres, cette polysémie est aussi un facteur de flou sémantique. Les motifs de la protection de la diversité biologique sont, eux aussi, très divers. Ils renvoient, d'une part, à des considérations écologiques et, en particulier, au rôle fonctionnel de la biodiversité, lequel fait l'objet de vives discussions au sein des SDV et, d'autre part, à des systèmes de valeurs humaines. D'un point de vue économique, on dispose de peu de chiffres et, le plus souvent, ce n'est pas la valeur de la diversité biologique proprement dite qui est mesurée, mais les services rendus par cette dernière à la société à travers un ensemble d'activités reposant sur l'exploitation de ressources biologiques (pêcheries, écotourisme...). D'un point de vue éthique, la diversité des objets que recouvre la biodiversité autorise des compromis entre les principales éthiques environnementales existantes (utilitarisme, biocentrisme, écocentrisme). Dans le domaine des mesures prises pour protéger la diversité biologique, on peut considérer deux grands types d'objets : le génie écologique, utilisé pour la conservation ou la réintroduction d'espèces jugées prioritaires, et les politiques publiques qui portent davantage sur la protection de territoires (par exemple, celles mises en œuvre dans le cadre de Natura 2000). Si les catégories du «naturel» et de l'« artificiel » sont travaillées au travers de ces actions, elles le sont de manière différenciée selon les champs scientifiques. Ainsi, la figure de l'ingénieur est prégnante chez les naturalistes, ce choix les mettant dans la position délicate de se référer à une éthique professionnelle qui a une obligation de résultats. En revanche, c'est celle du médiateur que retiennent volontiers les chercheurs des SHS, c'est-à-dire une personnalité capable de faire des bricolages institutionnels dans le cadre des scènes locales de négociation et de transaction autour des espaces et des objets biologiques qui y sont associés.

\section{Enjeux politiques et socio-économiques : biodiversité et droits de propriété}

Les droits de propriété sont des objets complexes qui ne relèvent pas d'une seule discipline puisqu'ils présentent de multiples dimensions sociales, juridiques, économiques, politiques et culturelles. Les réflexions à mener sur ces instruments de régulation sont primordiales puisque, depuis la conférence de Rio, la politique internationale de régulation relative à la biodiversité entend faire jouer un rôle central aux droits de propriété, en espérant qu'ils concilient la recherche d'avantages financiers et la protection de l'environnement. La Convention sur la diversité biologique (CDB) a ainsi reconnu trois types de droits de propriété : le droit souverain des États à exploiter leurs propres ressources biologiques, les droits de propriété intellectuelle (dont les brevets) détenus par les firmes et certains organismes publics et les droits des communautés autochtones et locales. À charge pour ces différents acteurs de négocier entre eux, en se référant à ces droits et en trouvant un consensus sur les conditions d'accès, de collecte et de rétribution des avantages industriels qui proviendraient de l'exploitation des ressources biologiques. Quinze ans après Rio, les rêves d'eldorado se sont dissipés : le nombre de contrats de bioprospection signés est beaucoup plus faible qu'escompté. L'inégalité entre les parties contractantes - firmes multinationales, d'un côté, pays du Sud et communautés locales, de l'autre - fait qu'il y a peu de versement financier pour des droits d'entrée ou de collecte d'échantillons biologiques ; les savoirs autochtones sont, eux aussi, peu rétribués. Les sommes concernées sont trop faibles pour être vraiment incitatives en matière de protection d'espaces riches en biodiversité. Enfin, un des problèmes majeurs réside dans la tension existante, pour ne pas dire la contradiction, entre la nécessité de reconnaître la diversité des ressources symboliques, dont disposent les groupes humains dans leur rapport au vivant, et l'obligation qui leur est faite par les organismes internationaux et les intérêts dominants de s'exprimer au travers des droits de propriété et des droits de propriété intellectuelle.

\section{Rôle des Organisations non gouvernementales (ONG) dans la gestion de la biodiversité}

La notion de biodiversité a pris sens et forme grâce, entre autres, aux ONG présentes dans les forums internationaux ou dans des actions de gestion sur le terrain. Inversement, la consécration politique de la biodiversité a été un élément de légitimation des ONG. Les grandes ONG de la conservation de la nature forment, au niveau mondial, une sorte d'oligopole décisionnel et financier organisé autour des aires naturelles protégées. Présentes dans certaines institutions internationales, ces ONG ont des liens avec les États et les opérateurs commerciaux, tout en s'appuyant sur leurs propres antennes installées localement ou sur d'autres ONG plus petites, créées bien souvent par des élites locales en phase avec les valeurs et modes de vie des responsables des grandes ONG. Au cours des années 1980, ces dernières, devant 
faire face à une contestation grandissante de leurs actions, ont voulu se relégitimer en se situant au cœur de la problématique du développement durable. Les grandes ONG ont alors approfondi la rhétorique économique utilisée depuis quelques décennies déjà. Or, l'économie de la diversité biologique, une sous-discipline de la théorie économique dominante, considère qu'il convient de mettre en valeur la biodiversité en lui attribuant les caractéristiques habituelles des biens économiques (des droits de propriété et des prix). L'accent mis sur les ressources génétiques par la CDB a encore accentué ce réductionnisme économique : à l'image du pétrole, la biodiversité est conçue comme un réservoir de ressources qu'il faut gérer rationnellement. Les pays du Sud apportent la matière première vivante, les pays du Nord la technique pour l'exploiter et les infrastructures industrielles et commerciales pour en diffuser les produits. L'espoir - vite déçu - des ONG conservationnistes était que ce commerce international de ressources biologiques permette de financer les aires naturelles protégées. Grâce au flou sémantique qui l'entoure, la biodiversité permet aussi aux ONG de jouer sur d'autres registres plus politiques encore : la globalité du problème de son érosion et des actions à mener pour la protéger justifie le dépassement du champ politique national et de la responsabilité des États. Cela s'est traduit notamment par un rapprochement entre ONG de protection de l'environnement et organisations des communautés autochtones. $\mathrm{Si}$, pour faire passer leurs stratégies, les unes ont emprunté aux autres leurs discours, voire leurs modes d'action, les médiations n'en restent pas moins difficiles entre des univers et des groupes sociaux qui n'ont pas la même vision ontologique du monde. Enfin, la diversité est aussi une notion politique en phase avec les référentiels mondiaux de l'époque : elle trouve en particulier sa place dans un discours libéral toujours prompt à dénoncer les actions des pouvoirs publics en faveur de plus d'égalitarisme. Pour autant - et ce n'est pas le moindre des paradoxes les politiques de conservation n'ont guère changé sur le terrain avec l'avènement de la problématique de la diversité biologique.

\section{Bilan général}

L'action du comité SEDD doit être analysée dans le contexte institutionnel très défavorable dans lequel s'est trouvé le PEVS-2 durant son mandat, de 1999 à début 2003, à savoir sa perte d'autonomie via son rattachement au département SDU, puis la volonté affichée par le $\mathrm{CNRS}^{12}$ de le supprimer en le remplaçant par un Institut national des sciences de l’Univers et de l'environnement

\footnotetext{
12 Avec l'aval de son organisme de tutelle, le ministère de la Recherche.
}

(INSU-E $)^{13}$. C'est dans cette ambiance délétère, où les jours du PEVS-2 étaient comptés et où la place des SHS dans le futur institut n'était pas garantie, qu'il convient de replacer le bilan de SEDD. En premier lieu, et dans la continuité des actions des comités ESDLT et SEAH, l'effort de structuration des équipes de recherche en SHS travaillant sur les problématiques environnementales a été poursuivi et amplifié, notamment auprès des communautés d'historiens et d'archéologues. Il a permis de créer un noyau d'équipes fédérant des chercheurs habitués à travailler avec des spécialistes d'autres champs disciplinaires sur des projets définis en commun. La réussite et les acquis dans ce domaine ont été indéniables, mais ils sont restés fragiles car l'effort de programmation impliquant les SHS doit se faire dans la durée sous peine d'une désagrégation rapide du vivier d'équipes ainsi créé. De plus, des difficultés d'ordre scientifique, académique et institutionnel persistent. En effet, l'idée que les problématiques environnementales sont d'abord des questions sociales, et qu'elles sont liées ensuite intimement à des questions naturalistes, et pas l'inverse, n'est pas encore partagée par tous. Par ailleurs, l'interdisciplinarité large, permettant d'aborder d'emblée la complexité d'un objet d'étude hybride à travers les points de vue des disciplines naturalistes et sociales, représente un réel défi scientifique, tant la formation académique tend à produire des "spécialistes », conditionnés à des raisonnements disciplinaires réductionnistes. D'un point de vue institutionnel, la promotion des chercheurs et surtout des universitaires engagés dans ce type de recherches, considérées comme annexes vis-à-vis du cœur de chaque discipline, reste toujours aléatoire. Il en est de même du recrutement au CNRS ou à l'Université de jeunes doctorants ayant travaillé sur de telles thématiques.

Concernant les avancées scientifiques, nous avons esquissé les apports marquants obtenus dans le cas des deux principales thématiques retenues par SEDD. Il est à noter que, dans le domaine de la diffusion des résultats, presque tous les programmes ont conduit à la publication, outre d'articles dans des revues disciplinaires, d'ouvrages de synthèse (Tableau), ce qui représente un net progrès par rapport aux comités précédents. Durant son mandat, SEDD a cherché à faire émerger de nouvelles questions de recherche. Il en a été ainsi des réflexions sur la gouvernance et la prospective qui ont donné lieu à des travaux malheureusement tardifs, car réalisés en fin de mandat. De réels progrès ont été enregistrés dans ces deux domaines, même si, à l'échelle du temps de la programmation scientifique du comité, ils ne peuvent être que modestes. Il est vrai que le manque de moyens humains et financiers et de temps en fin de programme ont empêché l'organisation d'un colloque de restitution des résultats obtenus par les équipes financées dans le cadre

\footnotetext{
13 Pour plus de détails, voir Muxart (2004) et Beck et al. (2008).
} 
Tableau. Activités scientifiques mises en place par le comité SEDD du PEVS-2 entre 1999 et 2002.

\begin{tabular}{|c|c|c|c|c|}
\hline $\begin{array}{l}\text { Nature } \\
\text { de l'action }\end{array}$ & Titre & Durée & $\begin{array}{l}\text { Informations } \\
\text { complémentaires }\end{array}$ & $\begin{array}{l}\text { Publications (auteurs, année, titre } \\
\text { et éditeur ou revue) }\end{array}$ \\
\hline \multirow[t]{2}{*}{$\begin{array}{l}\text { Appel } \\
\text { d'offres }\end{array}$} & $\begin{array}{l}\text { Histoire des interactions } \\
\text { sociétés-milieux }\end{array}$ & 1999-2002 & $\begin{array}{l}\text { Animations } \\
\text { complémentaires : } \\
\text { journées d'étude } \\
(25-26 / 10 / 01) \text {; séminaire } \\
\text { « Crises » et colloques } \\
\text { «Les fleuves aussi ont } \\
\text { une histoire» I et II. }\end{array}$ & $\begin{array}{l}\text { Muxart, T., Vivien, F.-D., Villalba, B., } \\
\text { Burnouf, J. (Eds), 2003. Des milieux } \\
\text { et des hommes : fragments d'histoires } \\
\text { croisées, Elsevier, Paris. }\end{array}$ \\
\hline & $\begin{array}{l}\text { Territoires, } \\
\text { environnement et nou- } \\
\text { veaux modes de ges- } \\
\text { tion : la gouvernance en } \\
\text { question }\end{array}$ & $2002-2004$ & $\begin{array}{l}\text { Pas de restitution des ré- } \\
\text { sultats en raison de la } \\
\text { suppression du PEVS-2, } \\
\text { mais publication d'ar- } \\
\text { ticles et d'ouvrages. }\end{array}$ & $\begin{array}{l}\text { Dubois, J., Maljean-Dubois, S. (Eds), } \\
\text { 2005. Natura 2000. De l'injonction } \\
\text { européenne aux négociations locales, } \\
\text { Paris, La Documentation française; } \\
\text { Scarwell, H.-J., Roussel, I., 2006. } \\
\text { Les Démarches locales de dévelop- } \\
\text { pement durable à travers les terri- } \\
\text { toires de l'eau et de l'air, Villeneuve } \\
\text { d'Ascq, Presses universitaires du } \\
\text { Septentrion; Bodiguel, M. (Ed.), } \\
\text { 2007. Gouvernance et partage de } \\
\text { l'eau : le bassin versant de Grand- } \\
\text { Lieu, Rennes, Presses universitaires } \\
\text { de Rennes; Goxe, A., Zuindeau, } \\
\text { B., 2008. Problèmes environnemen- } \\
\text { taux dans le bassin minier du } \\
\text { Nord-Pas-de-Calais : vers de nou- } \\
\text { velles gouvernances?, in Galochet, } \\
\text { M., Longuépée, J., Morel, V., Petit, } \\
\text { O. (Eds), L'Environnement: discours } \\
\text { et pratiques interdisciplinaires, Arras, } \\
\text { Artois Presses Université, 221-246; } \\
\text { Kirat, T., Torre, A. (Eds), 2008. Ter- } \\
\text { ritoires de conflits : analyses des muta- } \\
\text { tions de l'occupation de l'espace, Paris, } \\
\text { L'Harmattan. }\end{array}$ \\
\hline \multirow[t]{3}{*}{$\begin{array}{l}\text { Séminaires } \\
\text { de recherche }\end{array}$} & $\begin{array}{l}\text { Science, technique et en- } \\
\text { vironnement : l'écologie } \\
\text { industrielle en question }\end{array}$ & $2000-2002$ & $\begin{array}{l}\text { Responsable: } \\
\text { G. Rammuni, Centre P. } \\
\text { Léon (CNRS et Univer- } \\
\text { sité de Lyon 2), Lyon. }\end{array}$ & $\begin{array}{l}\text { Pas d'ouvrage de restitution, mais cf. } \\
\text { l'article de G. Rammuni in Lévêque, } \\
\text { C., van der Leeuw, S. (Eds), } 2003 . \\
\text { Quelles natures voulons-nous? Pour } \\
\text { une approche socio-écologique du champ } \\
\text { de l'environnement, Paris, Elsevier. }\end{array}$ \\
\hline & $\begin{array}{l}\text { Sociétés et ressources re- } \\
\text { nouvelables : temps et es- } \\
\text { paces des crises environ- } \\
\text { nementales }\end{array}$ & $2001-2003$ & $\begin{array}{l}\text { Responsables : C. Beck } \\
\text { (UMR 7941, MAE) } \\
\text { et Y. Luginbühl } \\
\text { (UMR LADYSS), Paris et } \\
\text { Nanterre. }\end{array}$ & $\begin{array}{l}\text { Beck, C., Luginbühl, Y., Muxart, T. } \\
\text { (Eds), 2006. Temps et espaces des crises } \\
\text { de l'environnement, Versailles, Quæ. }\end{array}$ \\
\hline & $\begin{array}{l}\text { Les ONG dans le champ } \\
\text { de la biodiversité }\end{array}$ & $2001-2003$ & $\begin{array}{l}\text { Organisé par un comité } \\
\text { de pilotage, secrétariat } \\
\text { C. Aubertin, IRD, } \\
\text { Orléans. }\end{array}$ & $\begin{array}{l}\mathrm{n}^{\circ} \text { spécial : "Les ONG et la gou- } \\
\text { vernance de la biodiversité », Re- } \\
\text { vue internationale des sciences sociales, } \\
\mathrm{n}^{\circ} 178 \text {, déc. 2003; Aubertin, C. } \\
\text { (Ed.), 2005. Représenter la nature? } \\
\text { ONG et biodiversité, Montpellier, IRD } \\
\text { Éditions. }\end{array}$ \\
\hline $\begin{array}{l}\text { Colloques } \\
\text { et journées } \\
\text { d'étude }\end{array}$ & $\begin{array}{l}\text { Journées «Biodiversité et } \\
\text { appropriation : les droits } \\
\text { de propriété en ques- } \\
\text { tion» }\end{array}$ & $20-21 / 06 / 00$ & $\begin{array}{l}\text { Responsables: } \\
\text { F.-D. Vivien (Univ. } \\
\text { Reims) et B. Guibert } \\
\text { (MEDD), Paris. }\end{array}$ & $\begin{array}{l}\text { Vivien, F.-D. (Ed.), 2002. Biodiversité } \\
\text { et appropriation : les droits de propriétés } \\
\text { en question, Paris, Elsevier \& NSS. }\end{array}$ \\
\hline
\end{tabular}


Tableau (suite).

\begin{tabular}{|c|c|c|c|c|}
\hline $\begin{array}{l}\text { Nature } \\
\text { de l'action }\end{array}$ & Titre & Durée & $\begin{array}{l}\text { Informations } \\
\text { complémentaires }\end{array}$ & $\begin{array}{l}\text { Publications (auteurs, année, titre } \\
\text { et éditeur ou revue) }\end{array}$ \\
\hline \multirow[t]{6}{*}{$\begin{array}{l}\text { Colloques } \\
\text { et journées } \\
\text { d'étude }\end{array}$} & $\begin{array}{l}\text { Journées «La prise en } \\
\text { compte du long terme et } \\
\text { de la prospective dans les } \\
\text { Zones Ateliers » }\end{array}$ & $29-30 / 03 / 01$ & $\begin{array}{l}\text { Co-organisé par SEDD } \\
\text { et NSS-Dialogues, } \\
\text { Meudon. }\end{array}$ & $\begin{array}{l}\text { Une série de comptes rendus dans } \\
\text { Natures Sciences Sociétés. }\end{array}$ \\
\hline & $\begin{array}{l}\text { Colloque «Valeurs et } \\
\text { représentations dans le } \\
\text { domaine de } \\
\text { l'environnement» }\end{array}$ & $14-15 / 05 / 01$ & $\begin{array}{l}\text { Co-organisé par SEDD, } \\
\text { NSS-Dialogues, MATE } \\
\text { et IFEN, Royaumont. }\end{array}$ & \\
\hline & $\begin{array}{l}\text { Colloque «Les fleuves } \\
\text { aussi ont une histoire } \\
\text { (I) : paléohydrologie des } \\
\text { quinze derniers millé- } \\
\text { naires » }\end{array}$ & $21-23 / 06 / 01$ & $\begin{array}{l}\text { Responsables: } \\
\text { J.-P. Bravard (Université } \\
\text { de Lyon 2) et M. Magny } \\
\text { (UMR Chrono-écologie, } \\
\text { Besançon), Motz. }\end{array}$ & $\begin{array}{l}\text { Bravard, J.-P., Magny, M. (Eds), } 2002 . \\
\text { Les Fleuves ont une histoire : paléoenvi- } \\
\text { ronnements des rivières et des lacs fran- } \\
\text { çais depuis } 15000 \text { ans, Paris, Errance. }\end{array}$ \\
\hline & $\begin{array}{l}\text { Colloque «Quelles } \\
\text { natures voulons-nous? } \\
\text { Quelles natures } \\
\text { aurons-nous?» }\end{array}$ & $12-14 / 11 / 01$ & $\begin{array}{l}\text { Organisé par le PEVS-2 } \\
\text { et ses différents comités } \\
\text { scientifiques, Lille. }\end{array}$ & $\begin{array}{l}\text { Lévêque, C., van der Leeuw, S. (Eds), } \\
\text { 2003. Quelles natures voulons-nous? } \\
\text { Pour une approche socio-écologique } \\
\text { du champ de l'environnement, Paris, } \\
\text { Elsevier. }\end{array}$ \\
\hline & $\begin{array}{l}\text { Colloque "Les fleuves } \\
\text { aussi ont une histoire (II) : } \\
\text { pratiques sociales et hy- } \\
\text { drosystèmes } \\
\text { fluviaux, lacustres et pa- } \\
\text { lustres des sociétés pré- } \\
\text { industrielles" }\end{array}$ & $8-10 / 04 / 02$ & $\begin{array}{l}\text { Responsables: } \\
\text { J. Burnouf (UMR } \\
\text { 7941, MAE) et Ph. } \\
\text { Leveau (Université } \\
\text { d'Aix-en-Provence), } \\
\text { Aix-en-Provence. }\end{array}$ & $\begin{array}{l}\text { Burnouf, J., Leveau, P. (Eds), } 2004 . \\
\text { Fleuves et marais, une histoire au croise- } \\
\text { ment de la nature et de la culture. Socié- } \\
\text { tés préindustrielles et milieux fluviaux, } \\
\text { lacustres et palustres : pratiques sociales } \\
\text { et hydrosystèmes, Paris, CTHS. }\end{array}$ \\
\hline & $\begin{array}{l}\text { Journées «Expertise et re- } \\
\text { cherche : les enjeux de } \\
\text { la directive-cadre euro- } \\
\text { péenne sur l'eau» }\end{array}$ & $29-30 / 04 / 02$ & $\begin{array}{l}\text { Organisé par le PEVS-2 } \\
\text { et ses différents comités } \\
\text { scientifiques, Paris. }\end{array}$ & $\begin{array}{l}\text { Bourgy, A., Lévêque, C. (Eds), } 2002 . \\
\text { Les Enjeux de la directive-cadre euro- } \\
\text { péenne de l'eau : synthèse des exposés et } \\
\text { débats, Antony, Cemagref Éditions. }\end{array}$ \\
\hline \multirow[t]{2}{*}{$\begin{array}{l}\text { Écoles } \\
\text { thématiques }\end{array}$} & $\begin{array}{l}\text { Méthodologies et enjeux } \\
\text { théoriques de la pros- } \\
\text { pective dans les projets } \\
\text { de recherche interdisci- } \\
\text { plinaires sur l'environne- } \\
\text { ment }\end{array}$ & $\begin{array}{l}28 / 09 / 01 \\
\text { au } 05 / 10 / 01\end{array}$ & $\begin{array}{l}\text { Organisé par le PEVS-2 } \\
\text { et ses différents comités } \\
\text { scientifiques, La Londe- } \\
\text { les-Maures. }\end{array}$ & $\begin{array}{l}\text { Mermet, L. (Ed.), 2003. Prospec- } \\
\text { tives pour l'environnement: quelles re- } \\
\text { cherches? quelles ressources? quelles } \\
\text { méthodes?, Paris, La Documentation } \\
\text { française; Mermet, L. (Ed.), } 2005 . \\
\text { Étudier des écologies futures: un chan- } \\
\text { tier ouvert pour les recherches pros- } \\
\text { pectives environnementales, Bruxelles, } \\
\text { PIE-Peter Lang. }\end{array}$ \\
\hline & $\begin{array}{l}\text { Biodiversité : quelles in- } \\
\text { teractions entre sciences } \\
\text { de la vie et sciences de } \\
\text { l'homme et de la société }\end{array}$ & $\begin{array}{l}07 / 10 / 12 \\
\text { au 11/10/02 }\end{array}$ & $\begin{array}{l}\text { Organisé par le CEFE } \\
\text { (Montpellier), le PEVS-2 } \\
\text { et SEDD, Porquerolles. }\end{array}$ & $\begin{array}{l}\text { Un compte rendu dans Natures } \\
\text { Sciences Sociétés (11, 3, 304-314); } \\
\text { Marty, P., Vivien, F.-D., Lepart, J., } \\
\text { Larrère, R. (Eds), 2005. Les Biodiver- } \\
\text { sités : objets, théories, pratiques, Paris, } \\
\text { CNRS Éditions. }\end{array}$ \\
\hline
\end{tabular}

de l'appel d'offres sur "La gouvernance en question », ce qui est tout à fait regrettable. Nous avons pu repérer un certain nombre d'ouvrages, de chapitres d'ouvrages ou de numéros spéciaux de revue parus ces dernières années, mais il ne s'agit là que d'une recension partielle.

Dans le domaine, alors très mouvant, de la réorganisation de la recherche environnementale au CNRS, vou-

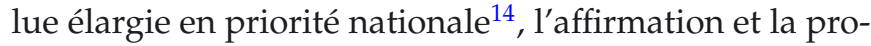
motion d'une interdisciplinarité large ont été au centre

14 Financement de l'INSU-E élargi aux autres organismes de recherche : Inra, Cemagref, IRD... de la stratégie d'action de SEDD. L'ardente obligation d'intégrer les problématiques des SHS dans les études environnementales et d'en faire a priori des questions sociales a fait l'objet d'un plaidoyer et d'une bataille institutionnelle de tous les instants, lorsqu'il est apparu clairement que le PEVS-2 n'allait pas être renouvelé. Beaucoup d'énergie a été ainsi consacrée à des interventions insistantes des représentants de SEDD auprès de la direction générale du CNRS et des directions scientifiques SDU et SHS. La question a également été portée sur la place publique (Muxart et Vivien, 2002), ainsi que dans 
le cadre des réunions de prospective visant à définir les questions vives et les politiques scientifiques à mettre en œuvre au sein de l'organisme.

Les résultats obtenus dans ce domaine, tant par SEDD que, plus généralement, par le directeur du PEVS-2 et le président du comité ZA, méritent d'être mis en perspective. Ils ont été performants quant à l'affichage ultérieur d'une interdisciplinarité large par la direction générale du CNRS et au maintien des ZA. Si la suppression du PEVS-2 n'a pas pu être empêchée, une division «Sociétés et environnements » a été instaurée dès 2003 au sein de $l^{\prime}$ INSU, en attendant sa transformation en INSU-E, et un responsable issu des SHS a été pressenti pour la diriger ${ }^{15}$. Une période confuse a suivi durant laquelle la création officielle de l'INSU-E a sans cesse été repoussée pour finalement échouer par suite d'oppositions externes au CNRS. Le rattachement à l'INSU a été abandonné au profit de la création, en 2006, d'un nouveau département scientifique autonome ${ }^{16}$ " Environnement et développement durable » (EDD) à vocation transversale, avec à sa tête un spécialiste de SDV. On notera avec une certaine ironie que cette appellation est conforme à l'une des propositions effectuées par SEDD ${ }^{17}$, à l'exclusion de la mention, pourtant fondamentale, de sociétés... Soulignons que cette réorganisation interne du CNRS revenait à pérenniser, sous une forme beaucoup plus durable, les activités contractuelles de près de 25 ans de l'ex-programme Environnement. Elle représentait donc une reconnaissance de l'importance de ses thématiques et aussi, de façon implicite, de l'intérêt de ses actions passées. Tout n'était pas réglé pour autant. Dès 2007, le CNRS a été soumis à une forte pression de son ministère de tutelle le contraignant à une réorganisation interne complète. En 2009, les départements scientifiques ont tous été supprimés et remplacés par des instituts autonomes rattachés à une direction générale. L'Institut écologie et environnement (INEE), dont la responsable est issue de SDV, a ainsi succédé au département EDD. L'affichage des SHS comme partie intégrante et essentielle des études sur l'environnement reste néanmoins flou. Comme en témoigne le projet scientifique de $1^{\prime} \mathrm{INEE}^{18}$, les objectifs sont, en premier lieu,

\footnotetext{
15 Pour des raisons personnelles, il a abandonné cette fonction très rapidement.

16 Au même niveau hiérarchique que les autres départements scientifiques, tels SDU, SDV, SHS...

${ }^{17}$ Le Comité SEDD avait proposé les dénominations «Environnements, sociétés et développement durable» ou «Interactions des sociétés avec leurs environnements ». Tandis que les deux responsables successifs du PEVS-1 et du PEVS-2 avançaient en haut lieu l'appellation «Interfaces milieuxécosystèmes-sociétés ".

18 Voir http://www.cnrs.fr/fr/organisme/instituts.htm : « [La mission de l'INEE est] de développer et de coordonner les recherches poursuivies dans les domaines de l'écologie et de l'environnement incluant la biodiversité et les relations hommesmilieux. »
}

naturalistes (écologie et biodiversité) et, dans un second temps, s'intéressent aux interactions des hommes (et non pas des sociétés) avec les milieux, ce qui rend possible l'intervention des sciences de l'homme. L'avenir dira si, à l'exception de l'économie, les sciences sociales (droit, sociologie, sciences politiques...) se sentiront concernées ou non par ce projet. L'ouverture viendra peut-être des recherches et problématiques définies dans les ZA qui seront maintenues. Soulignons pour terminer que l'appui aux thèses de l'interdisciplinarité large, intégrant les problématiques en SHS, est venu également de l'extérieur du CNRS et a été repris notamment dans le cadre des appels d'offres du ministère de l'Environnement.

\section{Remerciements}

Nous remercions vivement Corinne Beck pour sa relecture de cet article. Nous remercions aussi chaleureusement Marcel Jollivet pour ses encouragements et ses conseils qui ont fait suite à ses lectures attentives des différentes versions de ce texte.

\section{Références}

Beck, C., Muxart, T., Vivien, F.-D., 2008. Pour une approche interdisciplinaire de l'environnement. Les enseignements du " programme environnement » du CNRS, in Galochet, M., Longuépée, J., Morel, V., Petit, O. (Eds), L'Environnement : discours et pratiques interdisciplinaires, Arras, Artois Presses Université, 179-206.

Jollivet, M. (Ed.), 1992. Sciences de la nature, sciences de la société: les passeurs de frontières, Paris, CNRS Éditions.

Jollivet, M., 2001. Un exemple d'interdisciplinarité au CNRS : le PIREN (1979-1989), La revue pour l'histoire du CNRS, 4, 22-30.

Lévêque, C., 1998. La biodiversité : un avis d'écologue, Natures Sciences Sociétés, 6, 1, 37-40.

Lévêque, C., Pavé, A., Abbadie, L., Weill, A., Vivien, F.-D., 2000. Les zones ateliers, des dispositifs pour la recherche sur l'environnement et les anthroposystèmes, Natures Sciences Sociétés, 8, 4, 44-52.

Lévêque, C., Muxart, T., Abbadie, L., Weill, A., van der Leeuw, S., 2003. L'anthroposystème entité structurelle et fonctionnelle des interactions sociétés-milieux, in Lévêque, C., van der Leeuw, S. (Eds), Quelles natures voulons-nous? Pour une approche socio-écologique du champ de l'environnement, Paris, Elsevier, 110-129.

Muxart, T., Vivien, F.-D., 2002. Les recherches en environnement au CNRS : quelle interdisciplinarité veut-on?, Natures Sciences Sociétés, 10, 1, 66-68.

Muxart, T., 2004. La programmation des recherches interdisciplinaires en environnement au CNRS. Logique scientifique ou logique de pouvoir?, Natures Sciences Sociétés, 12, 310315.

Pavé, A., 2001. Deux programmes de recherche sur l'environnement dans les années 1990-1998 : le programme Environnement, puis le programme Environnement, vie et sociétés, La revue pour l'histoire du CNRS, 4, 32-46. 